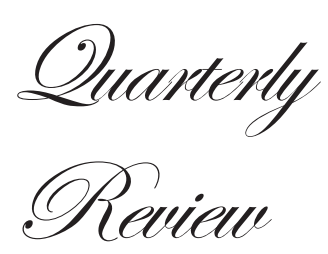

\title{
Status of confocal laser endomicroscopy in gastrointestinal disease
}

\author{
Jeremy Humphris, David Swartz, Brian J Egan, Rupert WL Leong
}

\begin{abstract}
Gastroenterology and Liver Services, Bankstown-Lidcombe Hospital, Sydney South West Local Health District and

The Faculty of Medicine, The University of New South Wales, Sydney, NSW Australia.

Confocal laser endomicroscopy (CLE) is an advanced imaging technique which combines conventional white light endoscopy (WLE) with an integrated or probe based confocal microscope. This allows microscopic examination of the surface epithelium and in vivo diagnosis during endoscopy. Established CLE applications include the diagnosis of Barrett's oesophagus, gastric intestinal metaplasia, coeliac disease and microscopic colitis. CLE can differentiate hyperplastic from adenomatous polyps in the colon and may obviate the need to biopsy all polyps at endoscopy. CLE is particularly helpful in surveillance endoscopy in inflammatory bowel disease where it has been shown to reduce the number of biopsies required and improve the detection of dysplasia. The future of CLE may be with new contrast agents to allow for molecular tagging and improved endoscopic diagnoses. The aim of this review is to describe the technology and techniques involved in CLE, and discuss the evolving applications in obtaining "virtual biopsy" throughout the GI tract.
\end{abstract}

KEYWORDS: Confocal, endomicroscopy, endoscopy, inflammatory bowel disease, Barrett's oesophagus, gastric intestinal metaplasia, gastric cancer, surveillance

\section{Introduction}

Conventional white-light endoscopy (WLE) has been utilised for decades to image the mucosa of the gastrointestinal tract. Technological advances over this time have allowed increasing optical resolutions, increasing miniaturisation of components and the development of endoscopic equipment which facilitate greater ease of access to the gastrointestinal tract for tissue sampling and treatment. Despite these advances, the underlying shortcoming of WLE has remained - the inability to ascertain any information other than gross mucosal morphological abnormalities. These technological advances have been paralleled by a greater understanding of the cellular and molecular changes that manifest as the abnormalities seen with WLE, as well as changes which may precede and subsequently develop into these abnormalities. Various technologies to complement WLE have therefore been developed to allow these previously unseen abnormalities to become apparent to the operator during endoscopic examination of the GI tract. Such technologies and techniques include narrow band imaging (NBI), chromoendoscopy, Raman spectroscopy, fluorescence endoscopy and optical coherence tomography. All these techniques have been striving to best obtain the holy grail of "optical biopsy" - that is the ability to 
obtain a tissue diagnosis in real-time, based on in-situ optical measurements, thereby obviating the need for tissue removal. Confocal laser endomicroscopy (CLE) is one such technology which allows real time "optical biopsy" during endoscopy. The technology and applications of CLE are rapidly evolving, and it is now a technique with increasing clinical relevance.

\section{Equipment}

Conventional confocal microscopy is a bench-top microscope that utilizes a spatial pinhole to eliminate out-of-focus light. As such, only the light within the focal plane of interest can be detected, enhancing the image quality and resolution. Using specific fluorescent-labeled antibodies, histochemical structures can be additionally identified as coloured-tags on the magnified images. Incorporating the confocal laser microscope into a conventional endoscope platform has allowed microscopy to take place in vivo during endoscopy.

\section{Endoscopic confocal endomicroscopy}

The first clinically applicable platform developed by Pentax (Tokyo, Japan) and Optiscan (Notting Hill, Victoria, Australia) was a confocal microscope incorporated into the tip of a standard $12.8 \mathrm{~mm}$ diameter endoscope (Pentax EC 3870CIFK, Pentax, Tokyo, Japan). Using a blue laser wavelength of 488 $\mathrm{nm}$, this endoscope CLE (eCLE) has a variable image collection rate of 0.8 or 1.6 frames per second. The field of view obtained is $500 \mu \mathrm{m} \times 500 \mu \mathrm{m}$, resolution of 7 micrometers, and achieves operator-controlled depth of imaging from the mucosal surface down to a depth of $250 \mu \mathrm{m}$. The endoscope has standard air and water channels in addition to a $2.8 \mathrm{~mm}$ auxiliary working channel to allow application of topical contrast agents. The result is the ability to target microscopic imaging of desired areas identified by conventional WLE, with multi-level microscopic imaging of cross-sections of mucosa with resolution to the sub-cellular level. This platform is the endoscopic CLE (eCLE). ${ }^{1,2}$

\section{Probe based confocal endomicroscopy}

An alternative instrument (Cellvizio; Mauna Kea Technologies, Paris, France) is a probe based system (pCLE) that is inserted through the working channel of any standard flexible endoscope. There are numerous probes available with variable diameters and optical characteristics depending on sites (Table 1). The probe system has a fixed image plane depth varying between $55 \mu \mathrm{m}$ and $130 \mu \mathrm{m}$ depending on the probes. Lateral resolution which is a measure of the ability of the system to detect closely separated objects ranges between $1 \mu \mathrm{m}$ and $3.5 \mu \mathrm{m}$. Due to their small diameters, the advantage of pCLE is the imaging of sites that cannot be evaluated by eCLE including the biliary and pancreatic ducts. The disadvantages of the probe-based system include limited lifespan of only 20 procedures. This increases the maintenance cost of the system. ${ }^{3}$ The occupation of the endoscope working channel by the pCLE also means topical agents cannot be easily applied or forceps biopsies taken, however double channel endoscopes may overcome these limitations.

\section{Contrast agents}

The CLE systems require fluorescent contrast agents in order to image the mucosa. A laser light source at a specific wavelength ( $488 \mathrm{~nm}$ is the current standard) excites the contrast fluorophore, which then re-emits the light at a different wavelength. ${ }^{4,5}$ The light at the new wavelength can be recognised by the CLE photosensor. The two exogenous fluorophores primarily used during CLE are fluorescein and acriflavine. ${ }^{6}$

\section{Fluorescein}

Fluorescein is an exogenous fluorophore that is administered intravenously as fluorescein sodium $10 \%$. The small proportion of fluorescein that is not bound to albumin in the serum is able to diffuse into the tissue and stain tissue components down to

Table 1. Optical characteristics of confocal endomicroscopy devices

\begin{tabular}{|c|c|c|c|c|}
\hline Confocal endomicroscope & $\begin{array}{l}\text { Depths of imaging } \\
\qquad(\mu \mathrm{m})\end{array}$ & $\begin{array}{l}\text { Lateral resolution } \\
\qquad(\mu \mathrm{m})\end{array}$ & $\begin{array}{l}\text { Image acquisition } \\
\text { (frames per second) }\end{array}$ & $\begin{array}{l}\text { Field of view } \\
(\mu \mathrm{m})\end{array}$ \\
\hline Endoscope CLE & $0-250$ & 0.7 & $0.8-1.6$ & $500 \times 500$ \\
\hline GastroFlex & $70-130$ & 3.5 & 12 & $600 \times 600$ \\
\hline ColoFlex & $70-130$ & 3.5 & 12 & $600 \times 600$ \\
\hline GastroFlex $^{\mathrm{UHD}}$ & $55-65$ & 1 & 12 & $240 \times 240$ \\
\hline ColoFlex $^{\mathrm{UHD}}$ & $55-65$ & 1 & 12 & $240 \times 240$ \\
\hline Cholangioflex & $40-70$ & 3.5 & 12 & $325 \times 325$ \\
\hline
\end{tabular}




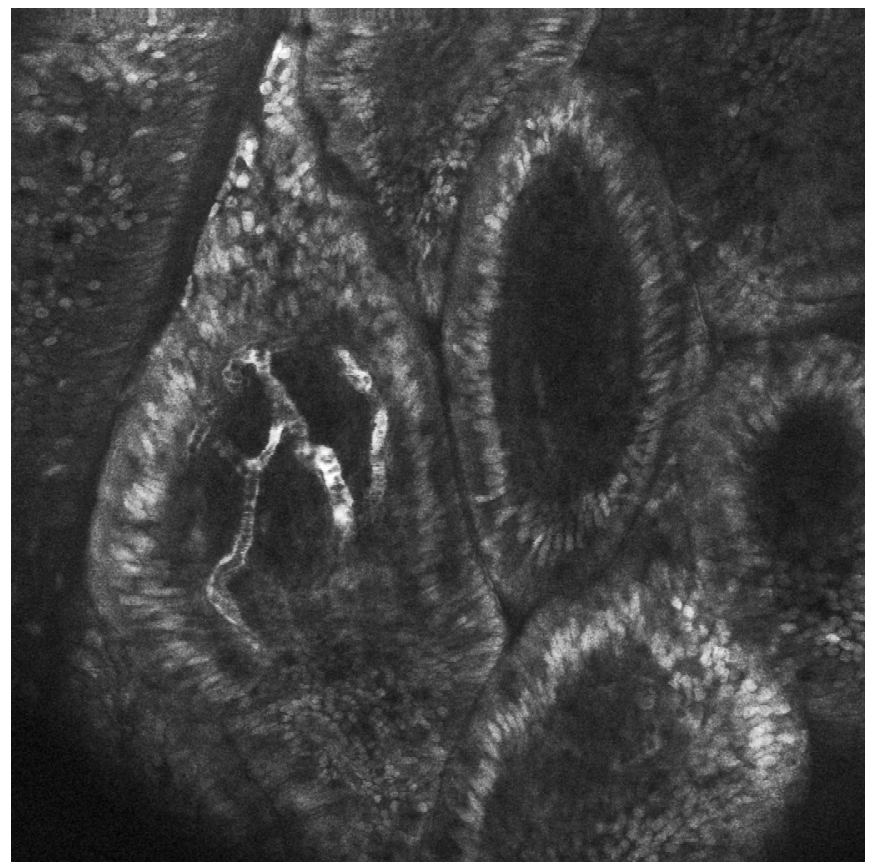

Figure 1: Confocal endomicroscopy of normal terminal ileum villi with goblet cells and blood vessels.

the lamina propria for up to $30-45$ minutes postadministration, ${ }^{1,2,6}$ however the image quality deteriorates significantly after 8 minutes. ${ }^{7}$ Cell nuclei and mucin remain unstained by fluorescein and are seen as dark areas when imaged by CLE. Fluorescein is able to highlight vasculature, red blood cells, lamina propria and intracellular spaces allowing vasculature and cellular architecture to be better appreciated (Figure 1). ${ }^{1,6}$ Long term experience with IV fluorescein comes from its application in ophthalmology with retinal fluorescein angiography. Serious adverse events such as acute hypotension and anaphylaxis have been noted but are exceedingly rare. Rates of any adverse reaction, most commonly nausea, transient skin discolouration or transient hypotension range from $1-6 \% .^{8-10}$ In the setting of CLE, very low rates of complications with no episodes of anaphylaxis have been published. ${ }^{11,12}$

\section{Acriflavine}

Acriflavine is an antiseptic derived from organic nitrogenous compounds. It is administered topically as acriflavine hydrochloride $0.05 \%$ to the mucosa of the GI tract. Its staining is restricted to acidic cell components, providing staining of cell nuclei which are not otherwise stained by fluorescein. The stain is able to penetrate to a depth of $100 \mu \mathrm{m}$ thereby aiding the assessment of mucosal cellular structures. It appears to be extremely safe according to published data and anecdotal experience. $^{6}$

\section{Technique}

The CLE endomicroscope is manipulated in the GI tract in a similar way as a regular flexible endoscope. Once the desired area of mucosa to be imaged is identified, the required contrast agent or agents can be administered topically through the working channel. Good quality imaging requires approximation of the endoscope tip to the identified area of interest, with or without gentle suction to allow the mucosa to abut the confocal microscope and reduce movement artefacts. Real-time microscopic images are seen on a screen, with the endoscopic view available on a second screen. Buttons on the scope handle allow the depth of imaging to be altered up and down to obtain different horizontal levels of cross-sectional image. Images can be captured and digitally stored by the system, allowing delayed examination of the images.

\section{Upper gastrointestinal applications Barrett's oesophagus}

Barrett's oesophagus (BO) is defined as the presence of intestinal-type oesophageal columnar metaplasia (IM). ${ }^{13} \mathrm{BO}$ often appears as red mucosa extending like a tongue in the distal oesophageal but its presence must be confirmed with biopsy and histological examination. BO can progress through a sequence of dysplasia and result in carcinoma. The annual incidence of cancer in patients with BO may range from 0.12 $0.5 \%$ per year. ${ }^{14-16}$ Endoscopic surveillance for detection of dysplasia and early neoplasia with systematic 4-quadrant biopsies every $2 \mathrm{~cm}$ is advocated in patients without suspected high-grade dysplasia (HGD) and at $1 \mathrm{~cm}$ intervals in patients with suspected HGD. ${ }^{17}$ This method of screening for dysplasia or early cancer is time-consuming, expensive, samples only a small surface area and there are small but real risks of obtaining tissue biopsies. These limitations have prompted the investigation of "red-flag" techniques to target biopsies, such as NBI, autofluorescence, chromoendoscopy and CLE.

Confocal endomicroscopy can accurately distinguish between squamous epithelium, gastric epithelium, Barrett's epithelium and Barrett's epithelium with neoplasia when compared to conventional light microscopy. At CLE each epithelial lining produces characteristic features in the epithelial cells, subepithelial capillaries and connective tissue. The initial description of eCLE in BO proposed a Confocal Barrett's Classification (CBC) system to predict in vivo histology. ${ }^{18} \mathrm{CLE}$ imaging of squamous mucosa shows squamous cells at high resolution and capillaries within single papillae. The number of 
papillae increases with epithelial damage as in erosive oesophagitis. Non-dysplastic BO is characterised by a regular villous-like epithelium with grey spots representing unstained goblet cell mucin in the upper parts of the mucosal layer and regular shaped subepithelial capillaries. Barrett's associated neoplasia is characterised by a distinctive polygonal cells with irregular apical and distal borders and has a uniform black appearance. The subepithelial capillaries are irregular in shape and may leak fluorescein producing a brighter signal in the lamina propria. Loss of the regular basement membrane integrity and the disruption of the villous epithelial structure strongly indicate high-grade dysplasia and/or early carcinoma. Differentiation between grades of dysplasia is not possible with current CLE techniques. In 63 patients with either long standing ( $>15$ years) reflux or surveillance for known BO, stored CLE images were able to predict the presence of $\mathrm{BO}$ and associated neoplasia with a sensitivity of $98.1 \%$ and $92.9 \%$ and a specificity of $94.1 \%$ and $98.4 \%$ respectively. ${ }^{18}$

Dunbar et al. validated the $\mathrm{CBC}$ in a double-blind randomized controlled trial of fluorescein-aided CLE and showed a positive effect on decision making. ${ }^{19}$ The study comprised of patients with $\mathrm{BO}$ undergoing routine surveillance or those referred with non-localised endoscopically unapparent high-grade dysplasia (HGD). Both groups underwent WLE with the standard 4-quadrant 1-2 cm random biopsy protocol and CLE with targeted biopsy with a 2-6 week interval between the procedures. This study attempted to emulate clinical practice with CLE images interpreted in real-time with no biopsy performed if no dysplasia was suspected on the images, and CLE optically-targeted biopsies were performed if dysplasia was suspected. The biopsy yield for dysplasia in the CLE group was $33.7 \%$ compared to $17.2 \%$ with standard endoscopy and random biopsies giving a difference in yield of $16.5 \%(\mathrm{p}=0.01)$. In the CLE group there was a significant reduction (almost $60 \%$ ) in the number of biopsies per procedure to detect neoplasia, with nearly two-thirds of the routine surveillance group not requiring a biopsy. The false-positive rate for suspected dysplasia at CLE was $64.3 \%$; therefore tissue biopsies are still required for a definitive diagnosis of dysplasia. CLE may also allow targeting areas of focal HGD for endoscopic therapy such as endoscopic mucosal resection..$^{20,21}$ In one study of 62 patients with suspected $\mathrm{BO}$ associated neoplasia, 22 were found to have flat HGD by CLE. These 22 underwent EMR with confirmed low grade dysplasia (LGD) in 6, HGD in 12 and no dysplasia in 4 giving a sensitivity of $94 \%$ and specificity of $50 \%$ for CLE-guided EMR for dysplasia. ${ }^{21}$
The pCLE has also been examined in patients with BO without areas suspicious for neoplasia on standard white-light endoscopy. Argon plasma coagulation was used to map the Barrett's segment and both CLE images and biopsies taken from a site immediately proximal. The resolution of the images obtained with the probe was not of sufficient resolution to assess cellular changes so the CBC was modified. The pCLE images detected HGD or early carcinoma by two blinded independent investigators with a sensitivity of $75 \%$ and specificity $57.9 \% .^{22}$

In addition to visualization of cellular and crypt architecture, CLE can image the subepithelial capillaries using IV fluorescein contrast. Angiogenesis is an essential step in carcinogenesis and is regulated by several growth factors of which vascular endothelial growth factor (VEGF) is considered the most important. ${ }^{23}$ The growth of a tumour is limited to 1-2 mm unless it can form new vasculature in and around the tumour. This process, which is termed "angiogenic switch", requires the tumour to produce several growth factors including VEGF. The angiogenic switch has been detected as an early event in the progression from Barrett's metaplasia to neoplasia. ${ }^{24}$ Angiogenesis due to VEGF overexpression produces vessels which are structurally different from normal vasculature. Tumour vasculature is tortuous, dilated and saccular with inconsistent branching and structurally frail walls producing leakage. ${ }^{25} \mathrm{CLE}$ visualizes the superficial mucosal layer capillaries because IV fluorescein bound to albumin remains predominantly intravascular. Using a software algorithm CLE can determine the microvessel density (MVD). Probe CLE determined that MVD was significantly higher in neoplastic versus non neoplastic Barrett's mucosa. ${ }^{26}$ A similar finding has been seen with oesophageal squamous cell carcinoma and gastric adenocarcinoma. ${ }^{27}$ Angiogenesis as an additional marker for neoplasia may increase the diagnostic accuracy of detecting neoplasia in Barrett's mucosa in the future.

Gastric intestinal metaplasia, gastric polyps and gastric cancer

There are no standard WLE features which accurately differentiate gastric intestinal metaplasia (GIM) from normal mucosa. Chromoendoscopy, magnification endoscopy, narrow band imaging (NBI) and enhanced magnification endoscopy can be used to identify GIM endoscopically. ${ }^{28}$ Accurate interpretation of the gastric pit pattern forms the basis of distinguishing between normal and diseased gastric mucosa. 
In the fundus the pits are composed of columnar cells with a round opening and are approximately uniform in size and shape. In the antrum the pits are continuous short rod-like pits with uniform length. Confocal endomicroscopy is able to visualize precisely the structure of the pit and can distinguish the pit pattern of normal mucosa from abnormal such as histologic gastritis, intestinal metaplasia and gastric adenocarcinoma. ${ }^{29}$ Intestinal metaplasia is a risk factor for intestinal-type gastric cancer. Under CLE it has 3 main features (a) a villous-like appearance with interstitium in the centre, (b) black appearing goblet cells and (c) columnar absorptive cells with a brush border. The sensitivity and specificity of these confocal features compared to histopathology is $98 \%$ and $95 \%$ respectively. ${ }^{30}$ The presence of goblet cells is a hallmark of GIM in conventional histology and its presence is the most sensitive marker in CLE. ${ }^{31}$ Early detection of gastric adenocarcinoma along with assessment of extent and depth of invasion is important. In 54 patients, 27 of who had early gastric cancer, CLE images obtained from the normal mucosa and from the cancerous lesions were interpreted by two pathologists and compared with H\&E stained transverse sections of the same sites. CLE images correlated well with the histological findings resulting in a sensitivity, specificity and accuracy of 81.8 $90.9 \%, 97.6 \%$ and $94.2-96.2 \%$, respectively, for the diagnosis of gastric adenocarcinoma. ${ }^{32}$ One problematic area with this technique is that good quality images are difficult to obtain due to movement artefacts caused by peristalsis, cardiac impulses and respiration. Confocal endomicroscopy allows immediate in vivo diagnosis of gastric mucosal neoplasia and pre-neoplasia. Using established diagnostic CLE features, diagnosis of intestinal metaplasia and gastric carcinoma can be made with a reliable level of interobserver agreement. ${ }^{33}$ In high risk Asian populations, CLE has potential clinical application in screening for gastric neoplasia and pre-neoplastic changes. ${ }^{34}$ CLE and EUS have been successfully utilised in the same endoscopic session for targeted biopsies and staging of gastric adenocarcinoma, lymphoma and GIST. 35 Gastric polyps are composed of fundic gland polyps, hyperplastic polyps and adenomas. Fundic gland polyps have no malignant potential while a small proportion of hyperplastic polyps show dysplastic changes or adenocarcinoma. Gastric adenomas are considered pre-malignant with an incidence of malignant transformation ranging from $4-70 \%$. In a study of 87 gastric polyps, the overall accuracy of CLE diagnosis of hyperplastic and adenomatous polyps during endoscopy was $90 \%$ and the overall accuracy of differentiating gastric hyperplastic polyps and adenomas by CLE after endoscopy was $97 \% .^{36}$

\section{Helicobacter pylori}

Helicobacter pylori (HP) are spiral-shaped, microaerophilic gram negative, urease-producing bacteria that adhere to the gastric epithelium. Helicobacter pylori induce chronic gastritis but more rarely cause peptic ulcer disease, gastric atrophy, intestinal metaplasia, gastric carcinoma, and gastric mucosalassociated lymphoma. The urease test sensitivity is $>90 \%$ and specificity is $>95 \%,{ }^{37}$ whereas histological analysis has a sensitivity of $93-96 \%$ and specificity of $98-99 \% .{ }^{38}$ Endoscopic CLE has shown promise in the detection of HP allowing immediate in vivo diagnosis. Acriflavine not only stains the gastric epithelial cells but also selectively stains HP which is seen as multiple white focal lesions at the surface and in deeper layers of the gastric epithelium. ${ }^{39}$ In a study of 118 consecutive patients with $H$. pylori infection-associated gastritis the sensitivity and specificity of CLE was $83 \%$ and $91 \%$ for the diagnosis of $H$. pylori infection, $93 \%$ and $95 \%$ for predicting glandular atrophy and $99 \%$ and $100 \%$ for diagnosing intestinal metaplasia, respectively. ${ }^{40}$

\section{Coeliac disease}

Coeliac disease (CD) is a gluten-sensitive enteropathy characterized by chronic inflammation of the proximal small intestinal mucosa induced immunologically by proteins of wheat, rye and barley. ${ }^{41}$ The resultant malabsorption and chronic inflammation may be complicated by nutritional deficiencies, infertility, malignancy and osteoporosis. Coeliac disease is diagnosed by characteristic small intestinal histological changes. A progression of mucosal injury first proposed by Marsh $^{42}$ and subsequently modified ${ }^{43,44}$ reflects the varying degrees of villous atrophy, inflammatory change, crypt lengthening and an increase in intraepithelial lymphocytes (IEL). Increased IEL alone (Marsh I) is not specific for CD. ${ }^{45}$ In the setting of suspected CD it is the number of villi, their characteristics (length, shape and presence/absence of transversal furrows) and the number of IEL that need to be well defined during CLE. ${ }^{46}$ False negatives in the histological diagnosis of $\mathrm{CD}$ include sampling error due to patchy distribution of mucosal changes, non-representative specimens, poor sampling quality, tangential sectioning, or if 
the specimen is not properly oriented (should be mounted villous side-up).

Endoscopic CLE has been evaluated in the diagnosis of $\mathrm{CD}$ in 31 patients comprising 6 with untreated $\mathrm{CD}, 11$ with treated CD and 14 controls. ${ }^{47}$ Superficial CLE images show the superficial surface villi whereas deep images show the basal villi. The eCLE images were paired with biopsies performed at the same sites and the independent interpretation of findings compared. Confocal villous atrophy was defined by the presence of 5 or fewer blunt shaped villi seen on superficial scans and crypt hypertrophy as one or more crypts on deep eCLE imaging. A CLE score (the Confocal Coeliac Score or $\mathrm{CCS}$ ) measuring CD severity was devised which was a ratio of images with definite features of $\mathrm{CD}$ against total images giving a number between 0 and 1, with a higher score representing greater severity. The accuracy of CLE in diagnosing CD was excellent with a sensitivity of $94 \%$ and specificity of $92 \%$ and correlated well with the Marsh grading. A CCS score of 0.06 or higher had a sensitivity of $94.1 \%$ and a specificity of $92.3 \%$ in making a true diagnosis of $\mathrm{CD}$. The site with the highest yield of CLE changes as indicated by the highest median CCS score was in the duodenal cap. Of the 17 cases with diagnosed CD, 16 were correctly diagnosed using CLE but only 13 had detectable histopathology changes on biopsy specimens. ${ }^{47}$ CLE may have the capability of providing immediate diagnosis or exclusion of $\mathrm{CD}$ and reduce costs ordinarily incurred with tissue processing and interpretation by a histopathologist. Another study recently reviewed the sensitivity and specificity of eCLE in the evaluation of established CD. ${ }^{48}$ In addition to villous atrophy, this study was able to identify IEL using CLE and maybe useful for diagnosing Marsh I CD. However, crypt hypertrophy had a lower sensitivity and specificity than Leong's study. This may be due to a different CLE definition of crypt hypertrophy requiring imaging of two or more crypts rather than just one, and not imaging for $\mathrm{CD}$ changes in the first part of the duodenum, proposed as the most sensitive site of detecting changes of CD. ${ }^{49}$

\section{Pancreatico-biliary}

The pancreatico-biliary tract remains one of the most challenging anatomical areas to diagnose disease. Early diagnosis of cholangiocarcinoma could allow treatment such as surgical resection or liver transplant in selected cases. Several methods such as endoscopic ultrasound, endoscopic retrograde cholangiopancreatography (ERCP) with tissue sampling, fluorescence in-situ hybridization (FISH) to detect chromosomal abnormalities and digital image analysis (DIA) to measure the intensity of dye-stained nuclei to quantify cellular DNA have been used to diagnose malignant strictures. ${ }^{50}$ There is emerging evidence for the usefulness of the pCLE in pancreatico-biliary pathology and its role in the further assessment of strictures or intraductal neoplasm in the pancreas and biliary tree. ${ }^{51,52}$ In a small study of fourteen patients with indeterminate biliary strictures who underwent probe based endomicroscopy a normal reticular pattern without loss of mucosal structures or irregular-appearing epithelium was a good predictor of a benign biliary stricture. ${ }^{50}$ The Miami classification has been developed to standardize probe based endoscopy indications and findings. ${ }^{53}$ A recent study of 102 patients with indeterminate pancreaticobiliary strictures showed that accuracy for combination of ERCP and pCLE was significantly higher compared with ERCP with tissue acquisition (90\% vs. $73 \%$; $\mathrm{p}=0.001) .{ }^{54}$ Further studies are required but $\mathrm{pCLE}$ may be an important adjunct to current modalities available in the diagnosis of malignant biliary strictures.

\section{Lower gastrointestinal applications Colorectal cancer}

The burden of colorectal cancer in Western countries is high. At present, the only demonstrated means of primary prevention of $\mathrm{CRC}$ is through colonoscopic detection and removal of adenomatous lesions before they develop into cancer. As there are no sufficiently accurate macroscopic means of determining polyp histology, all lesions must therefore be removed and examined histologically by a pathologist to assess for dysplasia. Under current recommendations, pathological diagnosis then dictates the frequency of further surveillance colonoscopies.

Attempts have been made to determine the confocal microscopic features of neoplasia and dysplasia in colon mucosal tissue. Ex vivo studies on a variety of neoplastic and non-neoplastic tissue using earlier confocal prototype systems attempted to define these characteristics. The two major features which identified normal and dysplastic or neoplastic tissue were visualisation of the cell nuclei characteristics, and architectural disruption of the mucosal epithelium and vasculature. ${ }^{55-57}$ The absence of these features appeared to be confirmatory of normal colonic tissue. ${ }^{55}$ A new classification for pCLE in the colon has been proposed and showed 'moderate' interobserver agreement for differentiating neoplasia from non-neoplastic tissue. The overall accuracy $(81 \%)$ for 
predicting neoplasia was acceptable and became excellent (94\%) when all study experts agreed. ${ }^{58}$ Further research refining and validating the classification is required.

The use of CLE as a means of further evaluating colonic lesions flagged as abnormal by the utilisation of chromoendoscopy has shown promising results. Kissliech et al ${ }^{59}$ studied 69 patients undergoing a screening or surveillance colonoscopy with the aid of methylene-blue chromoendoscopy to flag abnormal mucosa. Contrast-assisted CLE (C-CLE) of these areas were able to visualise any neoplastic features. These findings were correlated with formal histological assessment of biopsies of the areas. The presence of neoplastic changes was predicted by CLE with sensitivity of $97 \%$, specificity of $99 \%$ and accuracy of $99 \%$. The presence of irregular vasculature and fluorescein leakage on eCLE were additionally identified as indicative of neoplasia. The duration of the procedures was significantly longer than that of regular WLE. ${ }^{59}$ The ability of pCLE to distinguish between adenomatous and hyperplastic polyps has also been demonstrated. In a study of 116 polyps C-CLE accurately discriminated between adenomatous and nonadenomatous polyps and enabled evaluation of the degree of dysplasia during endoscopy. ${ }^{60}$

In a study of 130 polyps using CLE and narrow band imaging (NBI), the accuracy of pCLE and NBI was extremely high, approaching the accuracy of histopathology. Probe-based CLE demonstrated higher sensitivity in predicting histology of small polyps compared with NBI, whereas NBI had higher specificity ${ }^{61}$ Further development of CLE may obviate the need to sample all visualised polyps at colonoscopy. Residual neoplasia after EMR of colorectal lesions is common and CLE can significantly increase the sensitivity for detecting residual neoplasia after endoscopic mucosal resection compared with endoscopy alone. ${ }^{62}$

\section{Inflammatory bowel disease}

Much of the research into the clinical application of CLE has been in the area of neoplasia surveillance in inflammatory bowel disease colitis. CLE has also been used to identify intramucosal bacteria and classify disease activity in IBD. The risk of colorectal cancer in chronic colitis of greater than 8-10 years duration is well established, and surveillance protocols are established. These protocols recommend the biopsy of macroscopically abnormal areas of mucosa identified at WLE, as well as random, undirected biopsies at regular intervals along the colonic mucosa. The shortcomings of WLE in this setting are the time-intensive random biopsies required and the low yield of finding dysplasia.

Several studies have demonstrated the benefit of a combined chromoendoscopy-CLE examination to better detect early neoplastic changes. In a recent prospective randomised study, 161 patients with ulcerative colitis (UC) were randomised to conventional WLE, or colonoscopy with methylene blue chromoendoscopy and CLE examination of identified areas of mucosal abnormality. ${ }^{63}$ Combined chromoendoscopy and CLE detected 4.75-fold higher intra-epithelial dysplasia detection rate than conventional biopsy protocols ( 19 versus $4, \mathrm{p}=0.005$ ). Combined chromoendoscopy with CLE reduced the average number of biopsies taken by half ( 21.2 versus 42.2 , $\mathrm{p}=0.008$ ), whilst not increasing the mean procedure time. ${ }^{63}$

CLE has also been shown to be of benefit in distinguishing dysplasia-associated lesion or mass (DALM) from adenomalike masses (ALM) in the setting of chronic ulcerative colitis. These two lesion types cannot be accurately distinguished using WLE and require biopsy with potential repeat endoscopy for resection should an ALM be demonstrated. CLE using the modified Mainz criteria for in vivo diagnosis was able to differentiate with a high overall accuracy between DALM and ALM in 36 patients with a previously confirmed histological diagnosis. ${ }^{64}$ This potential use of CLE may allow identification of patients requiring referral for surgery or endoscopic lesion resection in a single procedure.

In a recent study, 73 consecutive patients with UC undergoing surveillance colonoscopy underwent CLE. Inflammation activity was first assessed by colonoscopy, then by CLE with a 4-grade classification of crypt architecture, as well as by analysis of microvascular alterations and fluorescein leakage. Targeted biopsy samples were taken for histological analysis and stored CLE images were subjected to post-CLE objective assessment. CLE seemed to be more accurate than conventional white-light endoscopy for evaluating macroscopically normal mucosa. More than half of the patients with normal mucosa seen on conventional white-light endoscopy showed acute inflammation on histology, whereas no patients with normal mucosa or with chronic inflammation seen on CLE showed acute inflammation on histology. Crypt architecture, microvascular alterations, and fluorescein leakage are promising markers in CLE evaluation of disease activity in IBD. ${ }^{65}$ Pouchitis and dysplasia may affect the pouch after restorative proctocolectomy in IBD patients. In a small study of 18 patients CLE was used to evaluate mucosal changes in 
the ileal pouch. At standard endoscopy, the signs of pouchitis were recorded in 7/18 (38.9\%) patients. At endomicroscopy, pathological features were found in 16/18 (88.9\%), villous atrophy in $15 / 18(83.3 \%)$, inflammation in $13 / 18(72.2 \%)$, ulceration in $3 / 18(16.7 \%)$, and colonic metaplasia in 12/18 $(67.7 \%)$. No dysplasia was observed. At histology, abnormalities were present in 17/18 (94.4\%): villous atrophy in $15 / 18(83.3 \%)$, inflammation in $17 / 18(94.4 \%)$, ulceration in $6 / 18$ $(33.3 \%)$, colonic metaplasia in $15 / 18(83.3 \%){ }^{66}$

The interaction of bacteria with the immune system within the intestinal mucosa plays a key role in the pathogenesis of IBD. CLE can image intramucosal bacteria in vivo and a significantly greater proportion of patients with Crohn's disease and ulcerative colitis have intramucosal bacteria than patients with a healthy intestine. Bacteria are found more frequently and with a wider distribution in patients with IBD than in patients with a normal intestine. ${ }^{67}$

\section{Microscopic colitis}

The exciting potential utility of CLE in the condition of microscopic colitis rests on the instant diagnosis of pathology in the absence of any macroscopic abnormality with conventional WLE. The thickened collagen layer beneath the basement membrane in collagenous colitis can be seen using eCLE. 68 The CLE appearances of a well developed "shell" around crypts have also been shown to correspond to the increased sub-epithelial collagen plate. ${ }^{69}$ In a series of 5 patients, demonstration of the thickened band of collagen at CLE correlated with formal pathological findings of biopsies taken from examined areas. ${ }^{68}$ This raises the prospect of instant diagnosis in cases of chronic watery diarrhoea without the need for forceps biopsy.

\section{Graft-versus-Host disease (GVHD)}

Graft-versus-Host disease is a significant complication of allogeneic haematopoietic stem cell transplantation (HCT). The distinction between acute and chronic GVHD has classically been determined by the time of onset post-transplantation (less or more than 100 days after HCT). ${ }^{70}$ This distinction now is no longer tenable with both acute and chronic forms occurring after and before 100 days and there can be overlapping features of both forms in the same patient. ${ }^{71,72}$ It commonly affects the skin, liver and the gut. Acute intestinal GVHD usually present with diarrhoea, whereas upper gastrointestinal GVHD causes anorexia, dyspepsia, nausea and vomiting. ${ }^{73}$ An early diagnosis by upper GI endoscopy or sigmoidoscopy and GI tract biopsies is imperative to enable an increase in immunosuppressive therapy. ${ }^{74}$ Patients with mild GVHD may have only very subtle endoscopic changes whereas those with severe GVHD usually have visible colitis. Often multiple biopsies are taken. Frequently these patients are thrombocytopenic or coagulapathic due to hepatic involvement with increased bleeding risk requiring platelet and/or clotting factor transfusion. Conventional histology using H\&E staining viewed with the light microscope is the accepted standard for diagnosing GVHD. It is important to obtain a histological diagnosis as diarrhoea is a common occurrence post-HCT and may be secondary to drug toxicity, opportunistic infections or antibiotic associated and to establish the GVHD activity. The histological hallmark of active GVHD although not pathognomonic for GVHD is the presence of apoptotic bodies in crypts the minimum number of which is not universally agreed but is usually $\geq 1 /$ piece. ${ }^{75}$ The endomicroscopic equivalent of an apoptotic cell is $100 \%$ nuclear staining with topical acriflavine. Bojarski and colleagues ${ }^{76}$ examined the endomicroscopic features and targeted biopsies with conventional histology of sigmoid and rectal biopsies ( 2 biopsies in each region) in 35 patients with acute diarrhoea post-HCT. Endoscopically only 6 showed signs of moderate to severe inflammation with erythema and ulceration. In 16 cases, endomicroscopy and histology detected no evidence of GVHD. In 19 cases there was evidence of GVHD on conventional histology comprising grade 1 in 10 , grade 2 in 3 , grade 3 in 2 and grade 4 in 4 . In all cases of grade 1 and 2 GVHD, endoscopy displayed no or minimal signs of inflammation. In 14 of these 19 cases the diagnosis of GVHD could be established by endomicrosopy during the procedure. In 5 of the 10 with histologically proven grade $1 \mathrm{GVHD}$, endomicroscopy showed single apoptotic cells within the crypt epithelium, while in the remaining 5 there were no changes seen. Other features seen at endomicroscopy for grades 2-4 include apoptosis of entire crypts, focal destruction of crypts, capillary leakage of fluoroscein and in severe cases complete crypt drop-out with a flat mucosa. In the 30 controls (15 infectious colitis, 15 ulcerative colitis) CLE showed inflammatory changes but no changes of GVHD. Therefore, for grades 2-4 there was a strong correlation between endomicrosopy and conventional histology with a sensitivity of $74 \%$ and specificity of $100 \% .{ }^{76}$ The early promise of CLE in GVHD may provide a real-time diagnosis of GVHD to be made allowing intensification of 
immunosuppression or at least targeting of areas of active disease with fewer biopsies and reduced risk of biopsy related complications.

\section{Functional imaging}

The gut epithelium acts as a barrier to intestinal contents. Complicating this task is the turnover of epithelial cells which results in each cell being replaced every 3-5 days leaving gaps and potential breakdown of the barrier function. In mice, epithelial gaps left by cell shedding have been demonstrated and shown to maintain the barrier function because they presumably contain an impermeable substance. ${ }^{77}$ Ex vivo fixed human intestinal specimens have not demonstrated these gaps. Using CLE in humans and mouse models these epithelial gaps have been demonstrated and can be clearly differentiated from goblet cells. In mice they maintain their barrier function (defined by exclusion of lucifer yellow entry into the epithelium) but show increased cell shedding and gap formation and loss of barrier function with exposure to tumour necrosis factor $\alpha$ (TNF $\alpha) .{ }^{78}$ In a study of 30 controls and 28 patients with IBD, the median epithelial gap densities for controls and patients with IBD were 18 and 61 gaps/1000 cells, respectively $(\mathrm{p}<0.001)$. Gap density however did not correlate with disease activity and patients with severe clinical disease also had lower median gap densities ( 37 vs. 90 gaps/1000 cells, $\mathrm{p}=0.04) .{ }^{79}$ It does seem likely that perturbation of these gaps is one mechanism leading to clinical manifestations in diseases such as inflammatory bowel disease and CLE may assist with diagnosis and assessing response to therapy. ${ }^{80}$

Targeted probes with affinity for certain peptide sequences specific for that particular tissue attached to a fluorescent tag may assist in the identification and diagnosis of specific pathologies. Hsiung et $\mathrm{al}^{81}$ developed a targeted heptapeptide that detects colonic dysplasia. Topical administration of the heptapeptide during pCLE detected dysplastic colonic cells with $81 \%$ sensitivity and $82 \%$ specificity. In vivo molecular imaging with specific targeting of VEGF is possible in tissue and can be performed in human colonoscopy using CLE. This could contribute to the identification of at risk lesions and potentially predict response to targeted treatment. ${ }^{82}$

\section{Conclusion}

Confocal endomicroscopy is a revolutionary optical technology that allows real-time in vivo visualisation of the mucosal layer at the cellular level during endoscopy. There is a high level of diagnostic accuracy for CLE and may have a significant effect on clinical decision-making. The diagnostic spectrum of CLE is currently expanding from screening and surveillance for dysplasia and neoplasia in the gastrointestinal tract, namely dysplasia in long-standing colitis, colorectal cancer and Barrett's oesophagus, to gastric cancer, squamous cell cancer and even non-neoplastic conditions such as coeliac disease and microscopic colitis. In addition, several other indications are being investigated such as MALT lymphoma and GVHD. CLE is likely to complement other "red-flag" techniques such as NBI which allows scanning of broader areas of mucosa, with areas of interest being interrogated by CLE. The concept of functional imaging sets CLE aside from conventional tissue biopsies, as ex vivo investigations loose information that may represent disease pathogenesis. The use of fluorescencelabeled antibodies may allow for specific targeting of pathology. Further technological developments and improvements may be possible to enhance the optical imaging capabilities further.

\section{References}

1. Kiesslich R, Goetz M, Vieth M, Galle PR, Neurath MF. Confocal laser endomicroscopy. Gastrointest Endosc Clinics N America. 2005;15:715-31.

2. Polglase AL, McLaren WJ, Skinner SA, Kiesslich R, Neurath MF, Delaney PM. A fluorescence confocal endomicroscope for in vivo microscopy of the upper- and lower-GI tract. Gastrointest Endosc. 2005;62:686-95.

3. ASGE Technology Committee, Kantsevoy SV, Adler DG, Conway JD, Diehl DL, Farraye FA, et al. Confocal laser endomicroscopy. Gastrointest Endosc. 2009;70:197-200.

4. Ell C. Improving endoscopic resolution and sampling: fluorescence techniques. Gut. 2003;52:iv30-3.

5. Stepp H, Stroka R, Baumgartner R. Fluorescence endoscopy in gastrointestinal diseases: basic principles, techniques, and clinical experiences. Endoscopy. 1998;30:379-86.

6. Hoffman E, Goetz M, Vieth M, Galle PR, Neurath MF, Kiesslich R. Confocal laser endomicroscopy: technical status and current indications. Endoscopy. 2006;38:1275-83.

7. Becker V, von Delius S, Bajbouj M, Karagianni A, Schmid RM, Meining A. Intravenous application of fluorescein for confocal laser scanning microscopy: evaluation of contrast dynamics and image quality with increasing injection-to-imaging time. Gastrointest Endosc. 2008;68;319-23.

8. Kwan AS, Barry C, McAllister IL, Constable I. Fluorescein angiography and adverse drug reactions revisited: the Lions Eye experience. Clin Experiment Ophthalmol. 2006;34:33-8.

9. Jennings BJ, Mathews DE. Adverse reactions during retinal fluorescein angiography. J Am Optom Assoc. 1994;65:465-71.

10. Kwiterovich KA, Maguire MG, Murphy RP, Schachat AP, Bressler NM, Bressler SB et al. Frequency of adverse systemic 
reactions after fluorescein angiography. Results of a prospective study. Ophthalmology. 1991;98:1139-42.

11. Wallace MB, Meining A, Canto MI, Fockens P, Miehlke S, Roesch T, et al. The safety of intravenous fluorescein for confocal laser endomiscroscopy in the gastro-intestinal tract. Aliment Pharmacol Ther. 2010;31:548-52.

12. Paramsothy S, Leong RW. Endoscopy: Fluorescein contrast in confocal laser endomicroscopy. Nat Rev Gastroenterol Hepatol. 2010;7:366-8.

13. Vakil N, van Zanten SV, Kahrilas P, Dent J, Jones R; Global Consensus Group. The Montreal definition and classification of gastroesophageal reflux disease: a global evidence-based consensus. Am J Gastroenterol. 2006;101:1900-20; quiz 1943.

14. Hvid-Jensen F, Pedersen L, Drewes AM, Sørensen HT, FunchJensen P. Incidence of adenocarcinoma among patients with Barrett's esophagus. N Engl J Med. 2011;365:1375-83.

15. Drewitz DJ, Sampliner RE, Garewal HS. The incidence of adenocarcinoma in Barrett's esophagus: a prospective study of 170 patients followed 4.8 years. Am J Gastroenterol. 1997;92:212-5.

16. Spechler SJ, Lee E, Ahnen D, Goyal RK, Hirano I, Ramirez F, et al. Long-term outcome of medical and surgical therapies for gastroesophageal reflux disease: follow-up of a randomized controlled trial. JAMA. 2001;285:2331-8.

17. Sampliner RE; Practice Parameters Committee of the American College of Gastroenterology. Updated guidelines for the diagnosis, surveillance and therapy of Barrett's esophagus. Am J Gastroenterol. 2002;97:1888-95.

18. Kiesslich R, Gossner L, Goetz M, Dahlmann A, Vieth M, Stolte $\mathrm{M}$, et al. In vivo histology of Barrett's esophagus and associated neoplasia by confocal laser endomicroscopy. Clin Gastroenterol Hepatol. 2006;4:979-87.

19. Dunbar KB, Okolo P 3rd, Montgomery E, Canto MI. Confocal laser endomicroscopy in Barrett's esophagus and endoscopically inapparent Barrett's neoplasia: a prospective, randomized, double-blind, controlled, crossover trial. Gastrointest Endosc. 2009;70:645-54.

20. Leung KK, Maru D, Abraham S, Hofstetter WL, Mehran R, Anandasabapathy S. Optical EMR: confocal endomicroscopytargeted EMR of focal high-grade dysplasia in Barrett's esophagus. Gastrointest Endosc. 2009;69:170-2.

21. Badreddine R, Wang K, Ganapathy A, et al. Confocal laser endomicroscopy (CLM) guided endoscopic mucosal resection in Barrett's esophagus with high grade dysplasia. Gastrointest Endosc. 2008;67:AB179.

22. Pohl H, Rösch T, Vieth M, Koch M, Becker V, Anders M, et al. Miniprobe confocal laser microscopy for the detection of invisible neoplasia in patients with Barrett's oesophagus. Gut. 2008;57:1648-53.

23. Hicklin DJ, Ellis LM. Role of the vascular endothelial growth factor pathway in tumor growth and angiogenesis. J Clin Oncol. 2005;23:1011-27.

24. Saad RS, El-Gohary Y, Memari E, Liu YL, Silverman JF. Endoglin (CD105) and vascular endothelial growth factor as prognostic markers in esophageal adenocarcinoma. Hum Pathol. 2005;36:955-61.
25. Meining A, Wallace MB. Endoscopic imaging of angiogenesis in vivo. Gastroenterology. 2008;134:915-8.

26. Becker V, Vieth M, Bajbouj M, Schmid RM, Meining A. Confocal laser scanning fluorescence microscopy for in vivo determination of microvessel density in Barrett's esophagus. Endoscopy. 2008;40:888-91.

27. Liu H, Li YQ, Yu T, Zhao YA, Zhang JP, Zhang JN, et al. Confocal endomicroscopy for in vivo detection of microvascular architecture in normal and malignant lesions of upper gastrointestinal tract. J Gastroenterol Hepatol. 2008;23:56-61.

28. Li CQ, Li YQ. Endomicroscopy of intestinal metaplasia and gastric cancer. Gastroenterol Clin North Am. 2010;39:785-96.

29. Zhang JN, Li YQ, Zhao YA, Yu T, Zhang JP, Guo YT, Liu H. Classification of gastric pit patterns by confocal endomicroscopy. Gastrointest Endosc. 2008;67:843-53.

30. Guo YT, Li YQ, Yu T, Zhang TG, Zhang JN, Liu H, et al. Diagnosis of gastric intestinal metaplasia with confocal laser endomicroscopy in vivo: a prospective study. Endoscopy. 2008;40:547-53.

31. In: Kiesslich R, Galle PR, Neurath MF, editors. Atlas of Endomicroscopy. Heidelberg: Springer Medizin Verlag; 2008.

32. Kitabatake S, Niwa Y, Miyahara R, Ohashi A, Matsuura T, Iguchi $\mathrm{Y}$, et al. Confocal endomicroscopy for the diagnosis of gastric cancer in vivo. Endoscopy. 2006;38:1110-4.

33. Yeoh KG, Salto-Tellez M, Khor CJ, Shah N, Ho KY. Confocal laser endoscopy is useful for in-vivo rapid diagnosis of gastric neoplasia and pre-neoplasia. Gastroenterology. 2005;128:A27-A27.

34. Nguyen NQ, Leong RW. Current application of confocal endomicroscopy in gastrointestinal disorders. $J$ Gastroenterol Hepatol. 2008;23:1483-91.

35. Gheorghe C, Iacob R, Dumbrava M, Becheanu G, Ionescu M. Confocal laser endomicroscopy and ultrasound endoscopy during the same endoscopic session for diagnosis and staging of gastric neoplastic lesions. Chirurgia (Bucur). 2009;104:17-24.

36. Li WB, Zuo XL, Zuo F, Gu XM, Yu T, Zhao YA, et al. Characterization and identification of gastric hyperplastic polyps and adenomas by confocal laser endomicroscopy. Surg Endosc. 2010;24:517-24.

37. Chey WD, Wong BC; Practice Parameters Committee of the American College of Gastroenterology. American College of Gastroenterologoy guideline on the management of Helicobacter pylori infection. Am J Gastroenterol. 2007;102:1808-24.

38. Howden CW, Hunt RH. Guidelines for the management of Helicobacter pylori infection. Ad Hoc Committee on Practice Parameters of the American College of Gastroenterology. Am J Gastroenterol. 1998;93:2330-8.

39. Kiesslich R, Goetz M, Burg J, Stolte M, Siegel E, Maeurer MJ, et al. Diagnosing Helicobacter pylori in vivo by confocal laser endoscopy. Gastroenterology. 2005;128:2119-23.

40. Wang P, Ji R, Yu T, Zuo XL, Zhou CJ, Li CQ, et al. Classification of histological severity of Helicobacter pylori-associated gastritis by confocal laser endomicroscopy. World J Gastroenterol. 2010;16:5203-10.

41. Rostom A, Murray JA, Kagnoff MF. American Gastroenterological Association (AGA) Institute technical review 
on the diagnosis and management of celiac disease. Gastroenterology. 2006;131:1981-2002.

42. Marsh MN. Gluten, major histocompatibility complex, and the small intestine. A molecular and immunobiologic approach to the spectrum of gluten sensitivity ('celiac sprue'). Gastroenterology. 1992;102:330-54.

43. Rostami K, Kerckhaert J, Tiemessen R, von Blomberg BM, Meijer JW, Mulder CJ. Sensitivity of antiendomysium and antigliadin antibodies in untreated celiac disease: disappointing in clinical practice. Am J Gastroenterol. 1999;94:888-94.

44. Oberhuber G, Granditsch G, Vogelsang H. The histopathology of coeliac disease: time for a standardized report scheme for pathologists. Eur J Gastroenterol Hepatol. 1999;11:1185-94.

45. Kakar S, Nehra V, Murray JA, Dayharsh GA, Burgart LJ. Significance of intraepithelial lymphocytosis in small bowel biopsy samples with normal mucosal architecture. Am J Gastroenterol. 2003;98:2027-33.

46. Trovato C, Sonzogni A, Ravizza D, Fiori G, Rossi M, Tamayo $\mathrm{D}$, et al. Celiac disease: in vivo diagnosis by confocal endomicroscopy. Gastrointest Endosc. 2007;65:1096-9.

47. Leong RW, Nguyen NQ, Meredith CG, Al-Sohaily S, Kukic D, Delaney PM, et al. In vivo confocal endomicroscopy in the diagnosis and evaluation of celiac disease. Gastroenterology. 2008;1356:1870-6

48. Günther U, Daum S, Heller F, Schumann M, Loddenkemper C, Grünbaum M, et al. Diagnostic value of confocal endomicroscopy in celiac disease. Endoscopy. 2010;42:197-202.

49. Leong RW. Confocal endomicroscopy in the evaluation of CD. Endoscopy. 2010;42:606; author reply 607.

50. Loeser CS, Robert ME, Mennone A, Nathanson MH, Jamidar P. Confocal endomicroscopic examination of malignant biliary strictures and histological correlation with lymphatics. J Clin Gastroenterol. 2011;45:246-252.

51. Meining A, Phillip V, Gaa J, Prinz C, Schmid RM. Pancreaticoscopy with miniprobe-based confocal laser-scanning microscopy of an intraductal papillary mucinous neoplasm (with video). Gastrointest Endosc. 2009:69;1178-80.

52. Meining A, Frimberger E, Becker V, Von Delius S, Von Weyhern $\mathrm{CH}$, Schmid RM, et al. Detection of cholangiocarcinoma in vivo using miniprobe-based confocal fluorescence microscopy. Clin Gastroenterol Hepatol. 2008:6:1057-60.

53. Wallace M, Lauwers GY, Chen Y, Dekker E, Fockens P, Sharma $\mathrm{P}$, et al. Miami classification for probe-based confocal laser endomicroscopy. Endoscopy. 2011;43:882-91.

54. Meining A, Chen YK, Pleskow D, Stevens P, Shah RJ, Chuttani $\mathrm{R}$, et al. Direct visualization of indeterminate pancreaticobiliary strictures with probe-based confocal laser endomicroscopy: a multicenter experience. Gastrointest Endosc. 2011;74:961-8.

55. Sakashita M, Inoue H, Kashida H, Tanaka J, Cho JY, Satodate $\mathrm{H}$, et al. Virtual histology of colorectal lesions using laser scanning confocal microscopy. Endoscopy. 2003;35:1033-8.

56. Inoue H, Cho JY, Satodate H, Sakashita M, Hidaka E, Fukami S, et al. Development of virtual histology and virtual biopsy using laser-scanning confocal microscopy. Scand J Gastroenterol Suppl. 2003;237:37-9.

57. Inoue H, Kudo SE, Shiokawa A. Technology insight: Laserscanning confocal microscopy and endocytoscopy for cellular observation of the gastrointestinal tract. Nat Clin Pract Gastroenterol Hepatol. 2005;2:31-7.

58. Kuiper T, van den Broek FJ, van Eeden S, Wallace MB, Buchner AM, Meining A, et al. New classification for probe-based confocal laser endomicroscopy in the colon. Endoscopy. 2011;43:1076-81.

59. Kiesslich R, Burg J, Vieth M, Gnaendiger J, Enders M, Delaney $\mathrm{P}$, et al. Confocal laser endoscopy for diagnosing intraepithelial neoplasias and colorectal cancer in vivo. Gastroenterology. 2004;127:706-13.

60. Sanduleanu S, Driessen A, Gomez-Garcia E, Hameeteman W, de Bruïne A, Masclee A. In vivo diagnosis and classification of colorectal neoplasia by chromoendoscopy-guided confocal laser endomicroscopy. Clin Gastroenterol Hepatol. 2010;8:371-8.

61. Shahid MW, Buchner AM, Heckman MG, Krishna M, Raimondo M, Woodward T,et al Diagnostic accuracy of probe-based confocal laser endomicroscopy and narrow band imaging for small colorectal polyps: a feasibility study. Am J Gastroenterol. 2012;107:231-9.

62. Shahid MW, Buchner AM, Coron E, Woodward TA, Raimondo M, Dekker E, et al. Diagnostic accuracy of probe-based confocal laser endomicroscopy in detecting residual colorectal neoplasia after EMR: a prospective study. Gastrointest Endosc. 2012;75:525-33.e1.

63. Kiesslich R, Goetz M, Lammersdorf K et al. Chromoscopyguided endomicroscopy increases the diagnostic yield of intraepithelial neoplasia in ulcerative colitis. Gastroenterology. 2007;132:874-82.

64. Hurlstone DP, Thomson M, Brown S, Tiffin N, Cross SS, Hunter MD. Confocal endomicroscopy in ulcerative colitis: differentiating dysplasia-associated lesional mass and adenoma-like mass. Clin Gastroenterol Hepatol. 2007;5:1235-41.

65. Li CQ, Xie XJ, Yu T, Gu XM, Zuo XL, Zhou CJ, et al. Classification of inflammation activity in ulcerative colitis by confocal laser endomicroscopy. Am J Gastroenterol. 2010;105:1391-6.

66. Trovato C, Sonzogni A, Fiori G, Ravizza D, Tamayo D, Botti F, et al. Confocal laser endomicroscopy for the detection of mucosal changes in ileal pouch after restorative proctocolectomy. Dig Liver Dis. 2009;41:578-85.

67. Moussata D, Goetz M, Gloeckner A, Kerner M, Campbell B, Hoffman A, et al. Confocal laser endomicroscopy is a new imaging modality for recognition of intramucosal bacteria in inflammatory bowel disease in vivo. Gut. 2011;60:26-33.

68. Kiesslich R, Hoffman A, Goetz M, Biesterfeld S, Vieth M, Galle PR, Neurath MF. In vivo diagnosis of collagenous colitis by confocal endomicroscopy. Gut. 2006;55:591-2.

69. Zambelli A, Villanacci V, Buscarini E, Bassotti G, Albarello L. Collagenous colitis: a case series with confocal laser microscopy and histology correlation. Endoscopy. 2008;40:606-8.

70. Glucksberg H, Storb R, Fefer A, Buckner CD, Neiman PE, Clift $\mathrm{RA}$, et al. Clinical manifestations of graft-versus-host disease in human recipients of marrow from HL-A-matched sibling donors. Transplantation. 1974;18:295-304.

71. Geeg HJ. How I treat acute GVHD. Blood. 2007;109:4119-26.

72. Filipovich AH, Weisdorf D, Pavletic S, Socie G, Wingard JR, Lee SJ, et al. National Institutes of Health consensus development 
project on criteria for clinical trials in chronic graft-versus-host disease: I. Diagnosis and staging working group report. Biol Blood Marrow Transplant. 2005;11:945-56.

73. Weisdorf DJ, Snover DC, Haake R, Miller WJ, McGlave PB, Blazar B, et al. Acute upper gastrointestinal graft-versushost disease: clinical significance and response to immunosuppressive therapy. Blood. 1990;76:624-9.

74. Cox GJ, Matsui SM, Lo RS, Hinds M, Bowden RA, Hackman $\mathrm{RC}$, et al. Etiology and outcome of diarrhea after marrow transplantation: a prospective study. Gastroenterology. 1994;107:1398-407.

75. Shulman HM, Kleiner D, Lee SJ, Morton T, Pavletic SZ, Farmer E, et al. Histopathologic diagnosis of chronic graft-versus-host disease: National Institutes of Health Consensus Development Project on Criteria for Clinical Trials in Chronic Graft-versusHost Disease: II. Pathology Working Group Report. Biol Blood Marrow Transplant. 2006;12:31-47.

76. Bojarski C, Günther U, Rieger K, Heller F, Loddenkemper $\mathrm{C}$, Grünbaum M, et al. In vivo diagnosis of acute intestinal graftversus-host disease by confocal endomicroscopy. Endoscopy. 2009:41;433-8.

77. Watson AJ, Chu S, Sieck L, Gerasimenko O, Bullen T, Campbell
F, et al. Epithelial barrier function in vivo is sustained despite gaps in epithelial layers. Gastroenterology. 2005;129:902-12.

78. Kiesslich R, Goetz M, Angus EM, Hu Q, Guan Y, Potten C, et al. Identification of epithelial gaps in human small and large intestine by confocal endomicroscopy. Gastroenterology. 2007:133;1769-78.

79. Liu JJ, Wong K, Thiesen AL, Mah SJ, Dieleman LA, Claggett $\mathrm{B}$, et al. Increased epithelial gaps in the small intestines of patients with inflammatory bowel disease: density matters. Gastrointest Endosc. 2011;73:1174-80.

80. Leong RW, Chang D, Merrett ND, Biankin AV. Taking optical biopsies with confocal endomicroscopy. J Gastroenterol Hepatol. 2009;24:1701-3.

81. Hsiung PL, Hardy J, Friedland S, Soetikno R, Du CB, Wu AP, et al. Detection of colonic dysplasia in vivo using a targeted heptapeptide and confocal microendoscopy. Nat Med. 2008;14:454-8.

82. Foersch S, Kiesslich R, Waldner MJ, Delaney P, Galle PR, Neurath MF, et al. Molecular imaging of VEGF in gastrointestinal cancer in vivo using confocal laser endomicroscopy. Gut. 2010;59:1046-55. 